\title{
Laboreal
}

Volume $15 \mathrm{~N}^{\circ} 1$ | 2019

Trabalho e cooperação

\section{Trabajo y cooperación: presentación del dossier}

Trabalho e cooperação: apresentação do dossiê

Travail et coopération: présentation du dossier

Work and cooperation: presentation of the dossier

Cirlene de Souza Christo, Jussara Brito, Letícia Pessoa Masson, Marcelo Figueiredo y Paulo César Zambroni-de-Souza

\section{OpenEdition}

\section{Journals}

Edición electrónica

URL: http://journals.openedition.org/laboreal/1046

DOI: $10.4000 /$ laboreal. 1046

ISSN: 1646-5237

Editor

Universidade do Porto

\section{Referencia electrónica}

Cirlene de Souza Christo, Jussara Brito, Letícia Pessoa Masson, Marcelo Figueiredo y Paulo César

Zambroni-de-Souza, «Trabajo y cooperación: presentación del dossier », Laboreal [En línea], Volume 15

N01 | 2019, Publicado el 01 julio 2019, consultado el 25 septiembre 2020. URL : http://

journals.openedition.org/laboreal/1046; DOI : https://doi.org/10.4000/laboreal.1046

Este documento fue generado automáticamente el 25 septiembre 2020.

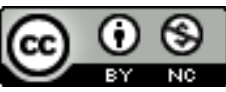

Laboreal está licenciado com uma Licença Creative Commons - Atribuição-NãoComercial 4.0

Internacional. 


\title{
Trabajo y cooperación: presentación del dossier
}

\author{
Trabalho e cooperação: apresentação do dossiê \\ Travail et coopération: présentation du dossier \\ Work and cooperation: presentation of the dossier \\ Cirlene de Souza Christo, Jussara Brito, Letícia Pessoa Masson, Marcelo \\ Figueiredo y Paulo César Zambroni-de-Souza
}

1 La propensión de los seres humanos para la cooperación tiene raíces evolutivas antiguas (Maturana; Rezepka, 2000). Si estos son el resultado de un proceso histórico y no el producto de un plan preestablecido, cabe destacar la importancia de la cooperación para su preservación y evolución a lo largo de ese proceso. Un recorrido en el que el trabajo, como un elemento constitutivo de nuestra especie, se revela un factor decisivo, ya sea a nivel filogénico u ontogénico.

2 Así como los otros mamíferos, los humanos, desde el inicio de su vida, no consiguen sobrevivir sin el amparo de terceros, como bien lo ha demostrado Winnicott (2006). El trabajo, por su turno, permite revivir, de una forma transformada, lo que se vivió en los teatros de la infancia (Dejours, 1996), de tal modo que es necesaria la presencia de terceros, no solamente la física, pero de alguien con quien se pueda de hecho cooperar. Solamente así será posible a cada uno desarrollar sus potencialidades, generando una zona de desarrollo (Vygotski, 1997; Clot, 2006) que a todos favorece. Por el contrario, la realización de la actividad con énfasis en la competición aporta desde luego un potencial de nocividad para quien la realiza, reforzando el individualismo negativo (Castel, 1995) y generando resultados deletéreos para los sujetos y para la sociedad.

\section{Cooperación, trabajo y salud}

3 Con el fin de obtener la maximización de los beneficios en el sector privado y una supuesta mejoría del desempeño en la esfera pública - en este caso, primordialmente desde la lógica de new public management (Hood, 1995) -, la organización del trabajo 
basada en el estímulo a la competición ha implementado algunas iniciativas de manera recurrente: la evaluación individual de desempeño, la búsqueda por metas cada vez más elevadas y la responsabilización del trabajador sin las condiciones adecuadas para la realización de su trabajo, entre otras medidas que obligan a hombres y mujeres a trabajar bajo exigencias cada vez más grandes.

En un entorno en el cual se diseminan de manera acentuada tanto relaciones contractuales como formas de gestión del trabajo flexibles, y a menudo precarias, se constata el agravamiento de un marco potencialmente nocivo para la salud de los trabajadores. Con la consolidación progresiva en el imaginario social de valores basados en el individualismo y en la competición, se delinea un escenario cuyo contenido dominante apunta hacia una división creciente de las categorías de representación y organización colectiva de los trabajadores, así como para la disolución de vínculos sociales y la fragmentación de los colectivos de trabajo. Además, el recrudecimiento de tales prácticas, lejanas de una perspectiva de gestión que contemple la gestión colectiva de las actividades, puede traer implicaciones nefastas para el trabajo, puesto que tienden a comprometer aquello que la cooperación y los colectivos tendrían de crucial (o de positivamente diferencial) para la eficacia de los procesos productivos.

Como defienden diversos autores (Yves Schwartz, Yves Clot, Christophe Dejours, Alain Wisner, entre otros), hay elementos en la actividad de trabajo - entre los cuales la cooperación ocupa un lugar central - que son indispensables para lograr la calidad y la productividad, sin desatender la salud y la seguridad. Un compromiso muy desafiador y que no puede perder de vista las agrupaciones poblacionales diversas que viven en localidades que circundan las organizaciones y sus instalaciones (Oddone; Marri; Gloria; Briante; Chiattella; \& Re, 1986). En ese sentido, cabe recordar las dos tragedias ocurridas en los años 2015 y 2019 en el estado brasileño de Minas Gerais, asociadas al rompimiento de embalses utilizados en el sector de minería y que resultaron en la muerte de más de 300 personas, entre trabajadores y residentes, además que ocasionar consecuencias ambientales catastróficas. Pese a que tales eventos no encuentran precedencia en el territorio brasileño y han adquirido una enorme repercusión internacional, hasta este momento no se ha constatado la debida responsabilización de los principales involucrados, en especial las empresas.

6 Se defiende en este dosier temático que la cooperación contribuye para la producción de reservas de alternativas (Schwartz, 2001; Schwartz, Fyad y Rufino, 2008) que pueden ofrecer espacio para elaborar y dar visibilidad a formas de organización del trabajo que no sean deletéreas y posibiliten el desarrollo de sociedades más justas (Schwartz, 2001; Schwartz, Fyad y Rufino, 2008; Dejours, 2015). En esa dirección, se destaca la importancia de la construcción de herramientas teórico-prácticas que puedan amparar proyectos de desarrollo que no tengan como parámetro valores meramente mercantiles, pero sí que recoloquen la actividad humana en su centro (Schwarts, 2018) $\mathrm{y}$, de esa forma, estén al servicio de la vida, de la salud, del medio ambiente y de la conquista de sentido en el trabajo.

7 Fue con la intención de contribuir para la discusión sobre la cooperación en el trabajo, y lo que adviene cuando esta está ausente o queda comprometida, que se ha convocado la comunidad académica para componer este dosier. ¿Cómo puede esta contribuir para la eficacia y la fiabilidad de los sistemas complejos? ¿De qué modo su fortalecimiento, así como la solidaridad en los colectivos de trabajo, se relaciona con los contenidos del trabajo, la salud y la seguridad de los(as) trabajadores(as)? ¿Qué implicaciones advienen 
para esta de las prácticas de gestión basadas en la competencia y prioritariamente orientadas por resultados y metas, valiéndose con frecuencia de los sistemas individualizados de evaluación del desempeño? ¿De qué manera esta comparece y/o necesita de ser construida en espacios informales de trabajo? ¿De qué modo esta se manifiesta o es deliberadamente desatendida horizontal o verticalmente en espacios tomados, o no, por la tercerización? ¿Cómo se operan las relaciones fuertemente intermediadas por herramientas con diversos niveles de desarrollo tecnológico?

\section{Lo que las investigaciones indican}

Los textos aquí presentes resultan de reflexiones surgidas de investigaciones empíricas en distintos medios de trabajo, en diferentes sectores productivos y de formas distintas de organización del trabajo, como enumeramos a continuación.

El artículo de Vanessa Barros y Fabiana Oliveira abre bien la discusión, contribuyendo para dar visibilidad al trabajo de catadores de materiales reciclables, cuya importancia desde el punto de vista social y medioambiental en el marco brasileño debe destacarse. El estudio toma como lócus dos cooperativas gestionadas por la lógica de la Economía Solidaria. En ese contexto, las autoras indican que la cooperación y la solidaridad son construcciones engendradas en el cotidiano de las relaciones de trabajo, que ocurren en medio de numerosas contradicciones y conflictos. Utilizando un enfoque fundamentado en la etnografía y en la investigación-acción, el estudio presentado analiza específicamente el trabajo de catadoras que actúan en la separación del material que llega a la sede de la empresa, considerada el corazón de los procesos de reciclado. Según las autoras, aunque las expresiones "cooperativa" y "solidaria" se encuentren en el principio del funcionamiento de esas empresas, la cooperación no es un elemento proporcionado a priori. En ese sentido, identifican la producción de acuerdos por las cooperadas que buscan atender, al mismo tiempo, las necesidades del colectivo y las especificidades de cada trabajadora. Tales acuerdos se configurarían desde la experiencia práctica y se basarían en valores no mercantiles como, por ejemplo, la consideración de las condiciones de cada uno en la gestión de la producción.

10 Seguidamente, presentamos dos artículos que tratan de la actividad en la minería: uno de la autoría de Fernanda Araújo, Vicente Nepomuceno y Denise Alvarez, otro escrito por João César Fonseca, José Newton de Araújo, Carlos Eduardo Vieira y Rodrigo Monteiro. El primero de estos, relata parte de una experiencia de investigación-acción en una minería de carbón, ubicada en el Sur de Brasil, que viene siendo gestionada por los propios trabajadores, también organizados bajo la forma de cooperativa. La (des)estructuración de los colectivos de trabajo es tomada por los autores como una vía de análisis de las variaciones de los índices de productividad de la empresa. Por medio de una investigación pautada por el Análisis Ergonómico del Trabajo (AET), han sido identificados factores organizacionales que interpelan la actividad de operadores de tractor que componen los equipos de producción en las frentes de exploración subterránea del carbón. La presión por la producción inmediata y la falta de reconocimiento del esfuerzo, acentuadas por la dificultad de mantener fijos los equipos de producción, ejercerían una fuerte influencia en la priorización (por parte de los operadores) del alcance de las metas individuales. Sin embargo, se constata que esto ocurre en detrimento de la totalidad y continuidad del ciclo de operaciones que 
componen el proceso productivo, indicando dificultades en la cooperación, pese la prescripción de que esta conste en el modelo de gestión adoptado.

11 En el segundo estudio, que se desarrolla en las instalaciones de una grande empresa de extracción de mineral de hierro con actuación en la región Norte de Brasil, se discute la actividad de mineros que actúan en pareja para operar una excavadora eléctrica. Tomando como base a la Clínica de la Actividad, los autores argumentan que, además del conocimiento técnico para la realización de esta actividad y en contraposición a la visión individualizante de la empresa, la dimensión colectiva de la actividad - instancia propiciadora de la cooperación en el trabajo - es un elemento estructural del proceso productivo. Ese elemento sería intrínseco al desarrollo del operador, involucrando tanto el trabajo en pareja como el hacer solidario con los demás miembros de los equipos de producción en la extracción mineral estudiada.

Como secuencia, figuran dos artículos que también evidencian la cooperación como un componente esencial para el desarrollo de las actividades de trabajo. Ambos remiten a la calidad de la prestación de servicios públicos.

13 Realizando una investigación en conjunto con magistrados, Ruri Giannini, Laerte Sznelwar, Seiji Uchida y Selma Lancman presentan materiales que demuestran que el trabajo de los magistrados de la Justicia del Trabajo del municipio de São Paulo, Brasil, pese a que posee un carácter aparentemente solipsista, exige el estabelecimiento de redes entre ellos. Dichas redes, aunque informales, se relevan necesarias para enfrentar aquello que rebasa lo que es exigido en el concurso para dicha función y lo que es proporcionado en la formación de la Escuela de Magistratura. Provistos de la perspectiva de la Ergonomía de la Actividad y de la Psicodinámica del Trabajo, señalan que, además de las formas de evaluación de los jueces que son básicamente cuantitativas e individuales, no hay mecanismos institucionales que potencialicen los intercambios entre pares y valoricen el retorno de la experiencia lo que, según los autores, puede comprometer la calidad y la productividad de los servicios.

Los análisis emprendidos por Yana Felix, Anísio Araújo y Thaís Máximo van en esta misma dirección, al demostrar que la cooperación es determinante para la calidad de la asistencia facilitada por los equipos del Servicio de Atención Móvil de Urgencia (SAMU) en un estado de la región Nordeste de Brasil. Mediante los aportes de la Psicodinámica del Trabajo y de la Sociología (en especial Bruno Maggi y Philippe Zarifian), se refieren a la demanda intrínseca de cooperación en el desarrollo de las actividades, que no se restringe a la establecida por los distintos trabajadores de la salud - conductores, médicos y enfermeros(as). De hecho, también enfatizan la necesaria cooperación de los usuarios (del propio paciente, así como de sus familiares y, en muchos momentos, de personas que estén próximas del lugar de atención) para la realización de una atención de esa naturaleza y la eficacia de los servicios prestados. Los autores indican que la comunicación es un componente esencial de la cooperación en ese contexto específico, que se desarrolla con la experiencia práctica y en la convivencia conjunta, sea en espacios formales o informales. La lectura del artículo nos remite a una reflexión sobre las graves implicaciones de eventuales obstáculos al establecimiento de una cooperación entre los protagonistas de las actividades desarrolladas en la atención en casos de urgencia y emergencia, tanto en lo que respecta a su éxito como en lo que concierne a la salud de los trabajadores involucrados.

15 Por fin, dos artículos demuestran el papel fundamental de la cooperación en la gestión de los riesgos y en las acciones de salud y seguridad en el trabajo. El primero, de 
Marcelle La Guardia y Francisco Lima, aborda la actividad de electricistas de manutención de líneas de transmisión de energía. El texto se centra en una controversia ocurrida en una concesionaria estatal del sector (en el Sudeste de Brasil) y que dividía las opiniones respecto a la reorganización (reducción) de los equipos de electricistas. La solución implicó una investigación que se valió del análisis ergonómico del trabajo (AET) y colocó el trabajo real en el centro del estudio, volviéndose la mirada hacia la actividad y las relaciones intersubjetivas, lo que posibilitó desvendar el modo como la cooperación y la confianza son construidas en el cotidiano y cómo actúan como base para la eficiencia de la gestión colectiva de los riesgos en situaciones complejas.

En una perspectiva afín, el texto de Pamela Astudillo y Carlos Ibarra parte de la hipótesis de que la precarización de las relaciones y condiciones de trabajo en Chile, que acompaña el modelo neoliberal, tiene debilitado el trabajo colectivo y la cooperación entre los(as) trabajadores(as). Aporta una discusión sobre la formación en ergonomía instituida en Chile, después de la adopción de una política nacional de salud y seguridad en el trabajo (SST) impulsada por el accidente de los 33 mineros en la mina de San José en 2010. A diferencia de lo que predominó, según los autores - una formación "técnica" fundamentada en la transmisión clásica de conocimientos por expertos - su investigación ha propuesto la formación-acción en ergonomía (de la actividad) y género, valorando el conocimiento y la experiencia de los trabajadores.

El estudio ha contemplado distintos sectores productivos (de la industria del salmón a los transportes, pasando por los servicios de salud), en los que se impartieron formaciones de corta duración por dirigentes sindicales y profesionales de SST. Para al artículo, os autores eligieron casos más estrechamente relacionados con trabajos en los que se realizaban pagos de incentivos por producción, indicando su asociación al elevado número de lesiones musculoesqueléticas y a la debilitación de las relaciones de cooperación, debido a la falta de cohesión y a la competición generadas por el sistema de trabajo. Para los autores, el análisis de la actividad en el contexto de formación ha permitido generar cambios en la representación del problema, partiendo, por ejemplo, de la comprensión de qua la cooperación entre compañeros puede representar el factor de protección a la SST.

\section{Invitación a la lectura}

Consideramos que los textos que componen el dosier evidencian la actualidad y la importancia del tema de la cooperación, indicando retos con relación a su mejor comprensión, tanto en las situaciones en que las actividades poseen un carácter aparentemente solipsista como en aquellas en que se presupone el trabajo colectivo. Los autores de los artículos también aportan cuestiones sobres situaciones presuntamente favorables a la cooperación, como en cooperativas, resaltando que ese aspecto del trabajo nunca está dado a priori, implicando la creación de reorganizaciones para la realización de las actividades. Además, incorporan como punto común el hecho de que una mirada cuidadosa y detenida sobre la actividad - partiendo de distintos métodos de análisis del trabajo y formación-acción - puede ser bastante fructífera para la instalación del diálogo sobre el trabajo y para el cuestionamiento de las normas y valores muchas veces impuestos por la organización del trabajo y que son deletéreas para la construcción de los lazos de cooperación. 
Si, por un lado, los artículos brindan elementos que indican los efectos negativos de los bloqueos a la cooperación en el trabajo para la eficiencia de los procesos productivos, para la gestión de los riesgos y para la salud de los trabajadores involucrados, por otro, revelan formas de cooperación tejidas en el desarrollo de las actividades, que se construyen y se consolidan con el tiempo, con la experiencia y el fortalecimiento de los vínculos.

Por fin, esperando de ese dosier contribuya para una mejor comprensión del trabajo en sus configuraciones en la contemporaneidad, lo invitamos, lector, a disfrutar de su lectura, subrayando que: si el individualismo y la competición se colocan como tendencias hegemónicas, nuevos caminos pueden ser construidos, puesto que "en lo que respecta a los sujetos humanos, nadie conoce ni conocerá integralmente las condiciones iniciales de su objeto de estudio, como también nadie conoce sus propias condiciones. Es por esa razón que la historia siempre nos reserva sorpresas" (Schwartz, 1992, p. 64).

\section{BIBLIOGRAFÍA}

Castel, R. (1995). Les métamorphoses de la question sociale: une chronique du salariat. Paris: Fayard.

Clot, Y. (2006). La fonction psychologique du travail. Paris: PUF.

Dejours, C. (2015). Le choix, Souffrir au travail n'est pas une fatalité. Montrouge: Bayard.

Dejours, Y. (1996). Uma nova visão do sofrimento nas organizações. In J.-F. Chanlat (Org.). O indivíduo na organização: dimensões esquecidas (pp. 150-173). São Paulo: Atlas.

Hood, C. (1995). The "new public management" in the 1980s: Variations on a theme. Accounting, Organizations and Society. 20(2-3), 93-109. https://doi.org/10.1016/0361-3682(93)E0001-W

Maturana, H.; Rezepka, Sima (2000). Formação humana e capacitação. Petrópolis: Vozes.

Oddone, I., Marri, G., Gloria, S., Briante, G., Chiattella, M., \& Re, A. (1986). Ambiente de trabalho: a luta dos trabalhadores pela saúde. São Paulo: Hucitec.

Schwartz, Y. (2018). Prefácio. In R. Di Ruzza, M. Lacomblez, \& M. Santos (Orgs). Ergologia, Trabalho, Desenvolvimentos (pp. 16-24). Belo Horizonte: Fabrefactum.

Schwartz, Y. (2001). Introduction aux IIIèmes Rencontres. In Association pour la Promotion des Recherches Interdisciplinaires sur le Travail - APRIT (Eds) Actes des IIIèmes Rencontres: Travail et civilisation: penser l'ouverture des espaces, des métiers, des pratiques (pp. 21-28). Université de Provence: Marseille.

Schwartz, Y. (1992). Travail et usage de soi. In Y. Schwartz (Org.). Travail et philosophie convocations mutuelles (pp. 43-66). Toulouse: Octarès.

Schwartz, Y. \& Adriano, R., Abderrahmane, F. (cols.) (2008). Revisitar a actividade humana para colocar as questões do desenvolvimento: projecto de uma sinergia franco-lusófona. Laboreal, 4(1), 10-22. http://laboreal.up.pt/revista/artigo.php?id=48u56oTV658223439657; 8:3872

Laboreal, Volume $15 \mathrm{~N}^{\circ} 1$ | 2019 
Vygotski, L. S. (1997). Pensée et Langage. Paris: La Dispute.

Winnicott, D. (2006). The family and individual development. London: Routledge.

\section{AUTORES}

\section{CIRLENE DE SOUZA CHRISTO}

Instituto de Psicologia da Universidade Federal do Rio de Janeiro. Av. Pasteur, 250 - Urca, Rio de Janeiro, RJ, CEP: 22290-902, Brasil; cirlenechr@gmail.com

\section{JUSSARA BRITO}

Fundação Oswaldo Cruz; Rua Leopoldo Bulhões 1480, Manguinhos, Rio de Janeiro, RJ, CEP: 21041-210, Brasil; jussara@ensp.fiocruz.br

\section{LETÍCIA PESSOA MASSON}

Centro de Estudos da Saúde do Trabalhador e Ecologia Humana, Escola Nacional de Saúde Pública Sérgio Arouca, Fiocruz; Rua Leopoldo Bulhões 1480, Manguinhos, Rio de Janeiro, RJ, CEP: 21041-210, Brasil; leticiamasson@ensp.fiocruz.br

\section{MARCELO FIGUEIREDO}

Escola de Engenharia - Departamento de Engenharia de Produção, Universidade Federal Fluminense Av. Passo da Pátria 156, São Domingos, Niterói, RJ, CEP: 22210-240, Brasil; marceloparada@uol.com.br

\section{PAULO CÉSAR ZAMBRONI-DE-SOUZA}

Programa de Pós-graduação em Psicologia Social - Departamento de Psicologia, Universidade Federal da Paraíba; Cidade Universitária, s/nº , Bairro Castelo Branco, João Pessoa, PB, CEP: 58051-900, Brasil; paulozamsouza@yahoo.com.br 